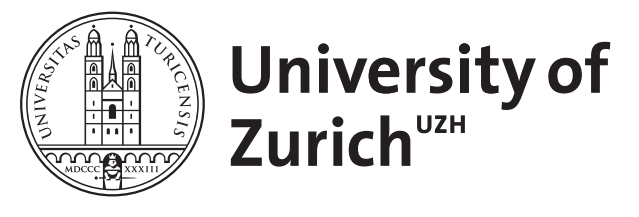

\title{
Tumor im Dünndarm bei Peutz-Jeghers-Syndrom
}

Friemel, J ; Varga, Z ; Weber, A

\begin{abstract}
Epithelial misplacement can be present in small bowel polyps of patients with Peutz-Jeghers syndrome and can mimic invasive malignancy. For recognizing epithelial misplacement, which can also have dysplastic changes, it is helpful to demonstrate the associated lamina propria and the lack of desmoplastic response. Intramural mucinous cysts are frequently seen in the setting of epithelial misplacement. Epithelverschleppung („epithelial misplacement“) kommt in Peutz-Jeghers-Polypen des Jejunum und Ileum vor. Sie kann zur Fallstrickdiagnose eines invasiven Adenokarzinoms verleiten. Hilfreich ist der Nachweis der erhaltenen Lamina propria um die verlagerten Epithelkomplexe, die selten auch Dysplasien aufweisen können. Epithelverschleppungen sind häufig mit intramuralem Schleim assoziiert.
\end{abstract}

DOI: https://doi.org/10.1007/s00292-012-1716-3

Other titles: Tumor in the small intestine in Peutz-Jeghers syndrome

Posted at the Zurich Open Repository and Archive, University of Zurich ZORA URL: https://doi.org/10.5167/uzh-73016

Journal Article

Published Version

Originally published at:

Friemel, J; Varga, Z; Weber, A (2013). Tumor im Dünndarm bei Peutz-Jeghers-Syndrom. Der Pathologe, $34(2): 170-172$.

DOI: https://doi.org/10.1007/s00292-012-1716-3 
Pathologe 2013 · 34:170-172

DOI 10.1007/s00292-012-1716-3

Online publiziert: 31. Januar 2013

๑) Springer-Verlag Berlin Heidelberg 2013

Redaktion

C. Kuhnen, Münster
J. Friemel · Z. Varga · A. Weber

Institut für klinische Pathologie, Universitätsspital Zürich, Schweiz

\section{Klinische Angaben}

Bei einer 38-jährigen Patientin mit bekanntem Peutz-Jeghers-Syndrom wurde 2 Monate zuvor ein muzinöses Adenokarzinom der Endozervix (Adenoma malignum) diagnostiziert. Aktuell fand sich ein verdächtiger Tumor im Jejunum.

\section{Morphologischer Befund}

Im $7 \mathrm{~cm}$ messenden Dünndarmresektat fand sich makroskopisch ein $2,5 \mathrm{~cm}$ großer Polyp mit intramuralen gelblich-schleimigen Zysten sowie eine fokal derb eingezogene Serosa. Histologisch zeigten sich im zunächst angefer- tigten Schnellschnitt unterhalb des Polypen Schleimseen und drüsige Epithelformationen in sämtlichen Darmwandschichten (• Abb. 1). Serosal aufgelagert fand sich Schleim. Die Epithelformationen hatten teils noch ihre ursprüngliche villöse Struktur, eine Lamina propria war im Schnellschnittpräparat nicht eindeutig nachweisbar. Da sich atypische Epithelabschnitte fanden, bestand der Verdacht auf eine „Low-grade“-Dysplasie.

\section{Fallstrickdiagnose}

- Adenokarzinom des Dünndarms auf dem Boden eines Peutz-Jeghers-Polypen
Abb. 1 - In die Darmwand verschleppte Epithelverbände und intramurale Schleimseen täuschen ein invasives Karzinom vor. Insert: $2,5 \mathrm{~cm}$ messender Polyp mit serosaseitigen Schleimauflagerungen in der Übersicht

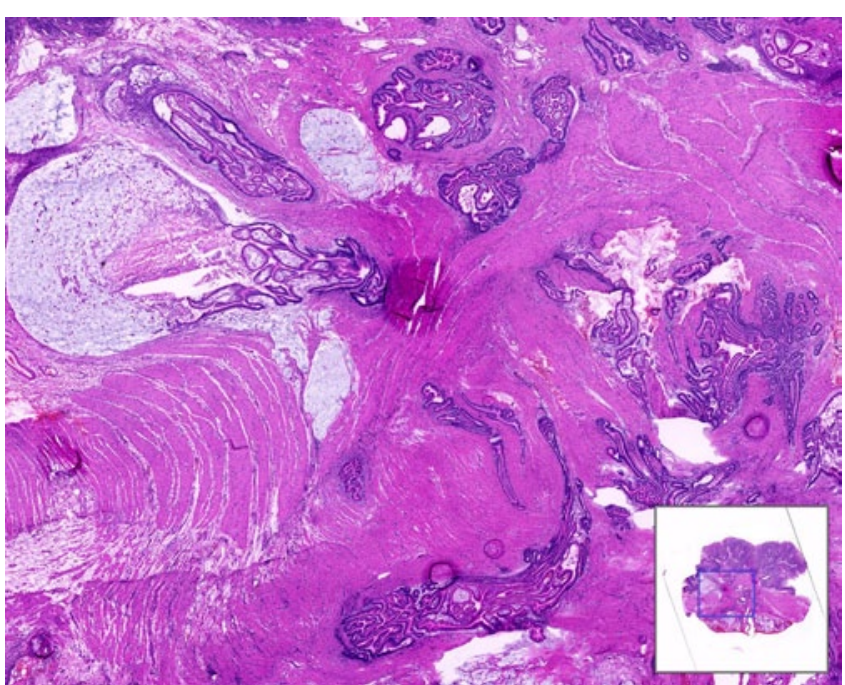




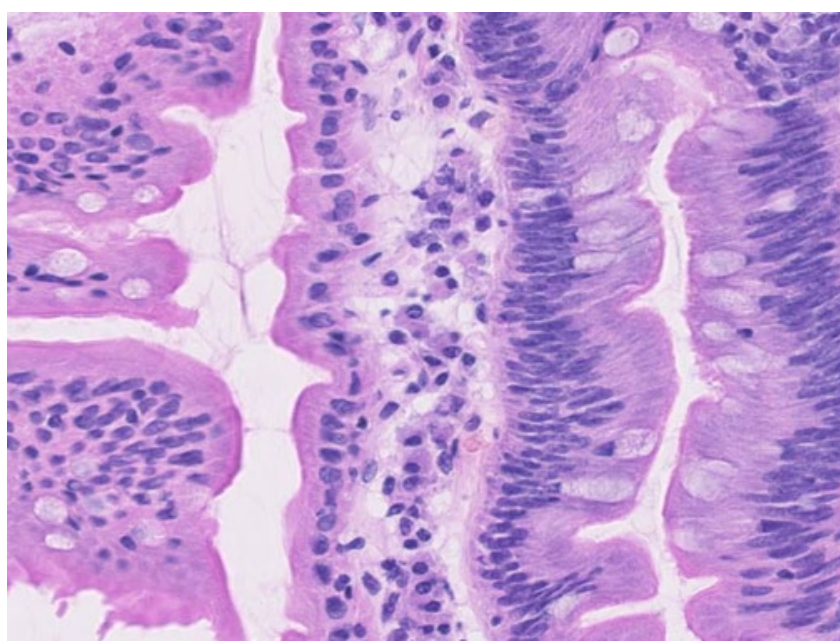

Abb. $2<$ Erhaltene Lamina propria um Epithelverbände, rechts Epithel mit „Lowgrade"-Dysplasie

\section{Verlauf und weitere Diagnostik}

Im routinemäßig aufgearbeiteten Präparat und mithilfe der Elastica-van-GiesonFärbung konnte die den Drüsenkomplexen teils noch anhaftende Lamina propria dargestellt werden. Weiterhin zeigte sich an den Epithelkomplexen ein erhaltener Bürstensaum und fokal eine Stratifizierung des Drüsenepithels mit Kernatypien, die als „Low-grade“-Dysplasie interpretiert wurden (• Abb. 2). Distinkt abgrenzbare Epithelanteile mit geringer Differenzierung waren nicht nachweisbar. Auch fand sich keine desmoplastische Stromareaktion um die Drüsen, und die Schleimseen zeigten keine isolierten dysplastischen Zellkomplexe („floating").

Der Tumor war CK7-negativ und CK20-positiv, was zusammen mit der Morphologie gegen eine Metastase des muzinösen Adenokarzinoms der Zervix sprach.
> Wie lautet Ihre Diagnose?

Durch den Nachweis der erhaltenen Lamina propria um die verlagerten Epithelformationen, das Fehlen einer desmoplastischen Stromareaktion und die erhaltene Differenzierung des Drüsenepithels wurde die Diagnose eines Peutz-Jeghers-Polypen mit Epithelverschleppung gestellt. Es lag keine echte Invasion und kein Adenokarzinom des Dünndarms vor.

\section{》) Diagnose: Benigner \\ Peutz-Jeghers-Polyp mit Epithelverschleppung}

\section{Diskussion}

Epithelverschleppung („,epithelial misplacement") von intestinalem Drüsenepithel in andere Darmwandschichten kann zur Fehldiagnose eines invasiven Adenokarzinoms führen und ist u. a. bei Kolonschleimhautadenomen [1] und hyperplastischen Polypen beschrieben worden [2]. Bei gestielten Polypen kann es zu Torsionen mit fokaler Blutung und Infarzierung kommen. Durch den entstehenden Defekt in der Lamina propria kann sich Epithel in die Submukosa verlagern.

In einer systematischen Analyse von 491 Peutz-Jeghers-Polypen konnte bei $10 \%$ eine Epithelverschleppung identifiziert werden [3]. In den analysierten Fällen kam die Verschleppung nur in Polypen des Ileum und Jejunum vor, nicht jedoch im Magen oder Duodenum. Die Autoren diskutieren, ob die in der Literatur angegebene Inzidenz von Peutz-Jeghers-assoziierten Adenokarzinomen des Magen-Darm-Traktes durch Epithelverschleppung zu hoch eingeschätzt wird. Selten kann das verschleppte Drüsenepithel Dysplasien aufweisen, was die Abgrenzung gegenüber einem malignen Tumor noch schwieriger macht [4].

Folgende Kriterien helfen bei der Unterscheidung von „,epithelial misplacement" und echter Invasion in Peutz-Jeghers-Polypen [3]:

- um die Drüsenkomplexe nachweisbare Lamina propria,

- histologisch normalerweise keine Atypie im verlagerten Drüsenepithel (selten Dysplasie),

- keine desmoplastische Stromareaktion um Epithelverbände,

- normaler Zellbesatz (Paneth-Zellen) und erhaltener Bürstensaum,

- Hämosiderinablagerungen und

- intramurale Schleimzysten.

\section{Korrespondenzadresse}

\section{Dr. J. Friemel}

Institut für klinische Pathologie, Universitätsspital Zürich

Schmelzbergstr. 12, 8091 Zürich

Schweiz

Juliane.friemel@usz.ch

Interessenkonflikt. Die korrespondierende Autorin gibt für sich und ihre Koautoren an, dass kein Interessenkonflikt besteht. 


\section{Literatur}

1. Muto T, Bussey HJ, Morson BC (1973) Pseudo-carcinomatous invasion in adenomatous polyps of the colon and rectum. J Clin Pathol 26:25-31

2. Yantiss RK, Goldman H, Odze RD (2001) Hyperplastic polyp with epithelial misplacement (inverted hyperplastic polyp): a clinicopathologic and immunohistochemical study of 19 cases. Mod Pathol 14:869-875
3. Shepherd NA, Bussey HJ, Jass JR (1987) Epithelial misplacement in Peutz-Jeghers polyps. A diagnostic pitfall. Am J Surg Pathol 11:743-749

4. Petersen VC, Sheehan AL, Bryan RL et al (2000) Misplacement of dysplastic epithelium in Peutz-Jeghers Polyps: the ultimate diagnostic pitfall? Am J Surg Pathol 24:34-39

\section{Hier kann auch Ihr Pitfall dar- gestellt werden}

Sind auch Sie schon einmal durch histopathologische oder zytologische Befunde in die Irre geleitet worden? Dann beschreiben Sie Ihren diagnostischen Fallstrick wie im nebenstehenden Beitrag und senden Ihren Pitfall an:

Prof. Dr. C. Kuhnen

Institut für Pathologie am

Clemenshospital Münster

Düesbergweg 128, 48153 Münster

kuhnen@patho-muenster.de 